S. Okada

Nagoya Math. J.

Vol. 94 (1984), 165-170

\title{
GENERALIZED MAILLET DETERMINANT
}

\author{
S. OKADA
}

\section{§1. Introduction}

In this paper, we shall study a generalization of the Maillet determinant. Let $p$ be an odd prime, and $G=(Z / p Z)^{*}$. We shall identify any integer and its image in $G$ if there is no fear of confusion. For any integer $a$, let $R(a)$ denote an integer satisfying

$$
R(a) \equiv a \bmod p, \quad 0 \leqq R(a)<p .
$$

Maillet studied the following determinant

$$
D_{p}=\operatorname{det}\left(R\left(a b^{-1}\right)\right)_{1 \leq a, b \leq(p-1) / 2}
$$

which is called the Maillet determinant and he raised the question whether $D_{p} \neq 0$ for all $p$. Carlitz-Olson [1] proved that the Maillet determinant is not zero by showing the following formula:

$$
D_{p}= \pm p^{(p-3) / 2} h^{-}
$$

where $h^{-}$is the first factor of the class number of $\boldsymbol{Q}\left(\zeta_{p}\right), \zeta_{p}$ the primitive $p$-th root of unity.

Carlitz considered a generalization of $D_{p}$ in [2]. We consider another generalization of the determinant $D_{p}$. Let $S$ be a subset of G. $S$ is called a CM-type if

$$
S \cup(-S)=G, \quad S \cap(-S)=\phi .
$$

Clearly $\{1,2, \cdots,(p-1) / 2\}$ is a CM-type. For any CM-type $S$, we define a determinant $D_{S}$ by

$$
D_{S}=\operatorname{det}\left(R\left(a b^{-1}\right)\right)_{a, b \in S} .
$$

We call $D_{S}$ the generalized Maillet determinant for $S$. Since $D_{S}=D_{-S}$, we may only consider CM-types which contain 1 .

Received March 22, 1983. 
Let $\chi$ be a Dirichlet character $\bmod p$. For any CM-type $S$, we define $c_{\chi}=c_{\chi}(S)$ by

$$
c_{\chi}=\sum_{a \in S} \chi(a) .
$$

Let $B_{1, \chi}$ denote the first generalized Bernoulli number. If $\chi$ is odd, $B_{1, \chi} \neq 0$. Therefore, we can define a rational number $A_{S}$ by

$$
A_{\bar{S}}=\frac{2}{p-1} \sum_{\chi . \text { odd }} c_{\chi} c_{\bar{\chi}} B_{1, \chi}^{-1} .
$$

Then we have

TheOREM. For any CM-type $S$ which contains 1, we have

$$
D_{S}=-\frac{1}{2}(-p)^{(p-3) / 2}\left(1+A_{S}\right) h^{-} .
$$

We shall prove this theorem and see the connection between our theorem and Carlitz-Olson's formula.

\section{$\S 2$. Proof of the theorem}

We need the following lemma, which is well-known as the Dedekind determinant [3]:

Lemma. Let $S$ be a CM-type, and $f$ be an odd function on $G$. Then the determinant $D(f)=\operatorname{det}\left(f\left(a b^{-1}\right)\right)_{a, b \in S}$ is independent of $S$, and

$$
D(f)=\sum_{\chi: \text { odd }} \frac{1}{2} \sum_{a \in G} \chi(a) f(a) .
$$

We define the determinant $D_{S}(x)$ as follows:

$$
D_{S}(x)=\operatorname{det}\left(R\left(a b^{-1}\right)+x\right)_{a, b \in S} .
$$

Since $R(a)-(p / 2)$ is an odd function, by Lemma

$$
\begin{aligned}
D_{S}\left(-\frac{p}{2}\right) & =\sum_{\chi: \text { odd }} \frac{1}{2} \sum_{a \in G} \chi(a)\left(R(a)-\frac{p}{2}\right) \\
& =\sum_{\chi: \text { odd }} \frac{p}{2} B_{1, \chi}=-\frac{1}{2}(-p)^{(p-3) / 2} h^{-} .
\end{aligned}
$$

And so, it suffices to show that

$$
\left[A_{S}\left(x+\frac{p}{2}\right)+\frac{p}{2}\right] D_{S}=\frac{p}{2}\left(1+A_{S}\right) D_{S}(x) .
$$


Now, it is clear that

$$
(p-1) R(a)=\sum_{x \in \hat{G}} \bar{\chi}(a) \sum_{b \in G} \chi(b) R(b),
$$

where $\hat{G}$ denotes the character group of $G$. If $\chi$ is not trivial, then

$$
\sum_{b \in G} \chi(b) R(b)=p B_{1, \chi} .
$$

Therefore,

$$
(p-1) R(a)=\frac{p(p-1)}{2}+p \sum_{x: \text { odd }} \bar{\chi}(a) B_{1, \chi}
$$

because $B_{1, \chi}=0$ for any non-trivial even character $\chi$. We define the rational number $A(a)$ as follows:

$$
A(a)=\sum_{x: \text { odd }} c_{\bar{z}} \chi(a) B_{1, x}^{-1} .
$$

Then it is clean that $A_{S}=\sum_{a \in S} A(a)$. For $b \in S$,

$$
\begin{aligned}
\sum_{a \in S} A & (a)\left(R\left(a b^{-1}\right)+x\right) \\
& =\sum_{a \in S} A(a)\left(x+\frac{p}{2}+\frac{p}{p-1} \sum_{x: \text { odd }} \bar{\chi}\left(a b^{-1}\right) B_{1, x}\right) \\
& =\frac{p-1}{2} A_{S}\left(x+\frac{p}{2}\right)+\frac{p}{p-1} \sum_{x: \text { odd }} \sum_{a \in S} A(a) \bar{\chi}\left(a b^{-1}\right) B_{1, x} .
\end{aligned}
$$

And then

$$
\sum_{x: \text { odd }} \sum_{a \in S} A(a) \bar{\chi}\left(a b^{-1}\right) B_{1, \chi}=\sum_{\chi: \text { odd }} \sum_{\psi: \text { odd }} c_{\bar{\psi}} B_{1, \psi}^{-1} \chi(b) B_{1, \chi} \sum_{a \in S} \psi \bar{\chi}(a) .
$$

We have

$$
\sum_{a \in S} \psi \bar{\chi}(a)=\left\{\begin{array}{cc}
\frac{p-1}{2} & \text { if } \chi=\psi \\
0 & \text { if } \chi \neq \psi
\end{array}\right.
$$

because $\psi \bar{\chi}$ is even and $S$ is a complete system of representatives of $G /( \pm 1)$. Hence

$$
\begin{aligned}
\sum_{\chi: \text { odd }} \sum_{a \in S} A(a) \bar{\chi}\left(a b^{-1}\right) B_{1, x} & =\frac{p-1}{2} \sum_{\chi: \text { odd }} c_{\bar{z}} \chi(b) \\
& =\frac{p-1}{2} \sum_{a \in S} \sum_{x: \text { odd }} \chi\left(a^{-1} b\right)=\left(\frac{p-1}{2}\right)^{2} .
\end{aligned}
$$

Consequently, 


$$
\sum_{a \in S} A(a)\left(R\left(a b^{-1}\right)+x\right)=\frac{p-1}{2}\left[A_{S}\left(x+\frac{p}{2}\right)+\frac{p}{2}\right]
$$

Therefore, there exists an integer $a_{i} \in S$ such that $A\left(a_{i}\right) \neq 0$.

Now we put the matrix $M(x)=\left(m_{a, b}\right)_{a, b \in S}$ as follows:

$$
m_{a, b}=\left\{\begin{array}{cl}
R\left(a b^{-1}\right)+x & \text { if } a \neq a_{i} \\
1 & \text { if } a=a_{i} .
\end{array}\right.
$$

Then, by some properties of matrices, we have

$$
A\left(a_{i}\right) D_{s}(x)=\frac{p-1}{2}\left[A_{s}\left(x+\frac{p}{2}\right)+\frac{p}{2}\right] \operatorname{det} M(x),
$$

and

$$
A\left(a_{i}\right) D_{s}=\frac{p(p-1)}{4}\left(1+A_{S}\right) \operatorname{det} M(0)
$$

Since $\operatorname{det} M(x)=\operatorname{det} M(0) \neq 0$ and $A\left(a_{i}\right) \neq 0$,

$$
\left[A_{s}\left(x+\frac{p}{2}\right)+\frac{p}{2}\right]=\frac{P}{2}\left(1+A_{s}\right) D_{s}(x) .
$$

This completes the proof of the theorem.

In the rest of this paper, we shall calculate $A_{S}$ in more convenient form, and show that Carlitz-Olson's formula follows easily from our theorem. Let $Z[G]$ be the group ring of $G$, and $Z[G]^{-}=\left\{\alpha \in Z[G] \mid \sigma_{-1} \alpha\right.$ $=-\alpha\}$ where $\sigma_{a}$ is the image in $G$ of an integer $a$. Let $S$ be a subset of $G$. We define the element $s(S)$ in $Z[G]$ by $s(S)=\sum_{\sigma \in S} \sigma$. We put the element

$$
\theta^{\prime}=\sum_{\sigma \in G} \frac{R(\sigma)}{p} \sigma^{-1} \quad \text { in } \boldsymbol{Q}[G],
$$

and the ideal of $Z[G]$

$$
\varphi^{\prime}=\theta^{\prime} Z[G] \cap Z[G]
$$

Then the Stickelberger element is defined by

$$
\theta=\sum_{\sigma \in G}\left(\frac{R(\sigma)}{p}-\frac{1}{2}\right) \sigma^{-1}=\varepsilon^{-} \theta^{\prime}
$$

where $\varepsilon^{-}=\frac{1}{2}\left(1-\sigma_{-1}\right)$. And the Stickelberger ideal is defined by

$$
\varphi=\theta Z[G] \cap Z[G]=\left\{\alpha \in \varphi^{\prime} \mid \sigma_{-1} \alpha=-\alpha\right\} .
$$


Moreover, by [4] we have the formula

$$
\left(Z[G]^{-}: \varphi\right)=h^{-} \text {. }
$$

For the CM-type $S_{0}=\{1,2, \cdots,(p-1) / 2\}$

$$
s\left(S_{0}\right)=\left(\sigma_{-1}+\sigma_{2}-1\right) \theta^{\prime},
$$

and so $s\left(S_{0}\right) \in \varphi^{\prime}$. Therefore, for any CM-type $S, h^{-}\left\{s(S)-s\left(S_{0}\right)\right\} \in \varphi$ because $s(S)-s\left(S_{0}\right) \in Z[G]^{-}$, and then $h^{-} s(S) \in \varphi^{\prime}$. Therefore,

$$
k s(S)=\theta^{\prime} \alpha
$$

for some $\alpha=\alpha_{s} \in Z[G]$ and some integer $k \mid h^{-}$. Then we have

Proposition. For any CM-type S,

$$
A_{S}=\frac{1}{k}\left(\sum_{\sigma \in S} n_{\sigma}-\sum_{\tau \in S} n_{\tau}\right)
$$

where $k s(S)=\theta^{\prime} \alpha, \alpha=\sum_{\alpha \in G} n_{\sigma} \sigma, n_{\sigma} \in Z$.

Proof. We extend a character $\chi$ to a function on $\boldsymbol{Q}[G]$ by

$$
\chi(\alpha)=\sum_{\sigma \in G} n_{\sigma} \chi(\sigma) \quad \text { for } \alpha=\sum_{\sigma \in G} n_{\sigma} \sigma \in \boldsymbol{Q}[G] .
$$

Then $c_{\chi}=\chi(s(S))$, and $B_{1, \chi}=\chi\left(\theta^{\prime}\right)$ for any non trivial character $\chi$. Hence

$$
k c_{\chi}=B_{1, \chi} \chi(\sigma)
$$

and so,

$$
A_{S}=\frac{2}{(p-1) k} \sum_{\chi: \text { odd }} c_{\bar{\chi}} \chi(\alpha) .
$$

For $\sigma \in G$,

$$
\sum_{\chi \cdot \text { odd }} \chi(\sigma)=\left\{\begin{array}{cl}
\frac{p-1}{2} & \text { for } \sigma=1 \\
-\frac{p-1}{2} & \text { for } \sigma=\sigma_{-1} \\
0 & \text { otherwise }
\end{array}\right.
$$

Therefore,

$$
\sum_{\chi: \text { odd }} c_{\bar{\chi}} \chi(\sigma)=\sum_{\chi: \text { odd }} \sum_{\tau \in S} \chi\left(\sigma \tau^{-1}\right)=\left\{\begin{array}{cc}
\frac{p-1}{2} & \text { for } \sigma \in S \\
-\frac{p-1}{2} & \text { for } \sigma \notin S
\end{array}\right.
$$


Consequently,

$$
A_{S}=\frac{1}{k}\left(\sum_{\sigma \in S} n_{\sigma}-\sum_{\tau \in S} n_{\tau}\right)
$$

This completes the proof.

By the proposition and the theorem, for $S_{0}$ we have Carlitz-Olson's formula.

\section{REFERENCES}

[1] L. Carlitz and F. R. Olson, Maillet's determinant, Proc. Amer. Math. Soc., 6 (1955), 265-269.

[2] L. Carlitz, A generalization of Maillet's determinant and a bound for the first factor of the class number, Proc. Amer. Math. Soc., 12 (1961), 256-261.

[ 3 ] S. Lang, Cyclotomic Fields, Springer-Verlag, G.T.M., 1978.

[4] K. Iwasawa, A class number formula for cyclotomic fields, Ann. of Math., 76 (1962), 171-179.

Department of Mathematics

Faculty of Science

Nagoya University

Chikusa-ku, Nagoya 464

Japan 